\title{
Monotone Interval Fuzzy Inference Systems
}

\author{
Yi Wen Kerk (), Kai Meng Tay ${ }^{\left({ }^{\circ}\right.}$, and Chee Peng Lim ()
}

\begin{abstract}
In this paper, we introduce the notion of a monotone fuzzy partition, which is useful for constructing a monotone zeroorder Takagi-Sugeno-Kang Fuzzy Inference System (ZOTSKFIS). It is known that a monotone ZOTSK-FIS model can always be produced when a consistent, complete, and monotone fuzzy rule base is used. However, such an ideal situation is not always available in practice, because a fuzzy rule base is susceptible to uncertainties, e.g., inconsistency, incompleteness, and nonmonotonicity. As a result, we devise an interval method to model these uncertainties by considering the minimum interval of acceptability of a fuzzy rule, resulting in a set of monotone interval-valued fuzzy rules. This further leads to the formulation of a Monotone Interval Fuzzy Inference System (MIFIS) with a minimized uncertainty measure. The proposed MIFIS model is analyzed mathematically and evaluated empirically for the Failure Mode and Effect Analysis (FMEA) application. The results indicate that MIFIS outperforms ZOTSK-FIS, and allows effective decision making using uncertain fuzzy rules solicited from human experts in tackling real-world FMEA problems.
\end{abstract}

Index Terms-Failure mode and effect analysis, monotone fuzzy partition, monotone interval fuzzy inference system, monotonicity, Takagi-Sugeno-Kang fuzzy inference system.

\section{INTRODUCTION}

$\mathbf{T}$ HE importance of a fuzzy inference system (FIS) that preserves the monotonicity property between its input and output [1]-[8] (hereafter denoted as a monotone FIS model) is widely studied and acknowledged [1]-[12]. Various useful mathematical conditions to satisfy the monotonicity property are available for different FIS models, e.g., Mamdani FIS [8], TSK-FIS [1], [9], [10], single-input rule module FIS [2], hierarchical FIS [3], and interval type-2 FIS [11]. The study in [8] indicates that product t-norm coupled with a consistent, complete, smooth, and monotone fuzzy rule base is required for constructing a monotone Mamdani FIS model. In addition, a fuzzy membership function (FMF) that satisfies specific mathematical conditions at the antecedent part, coupled with a consistent, complete, and monotone fuzzy rule base at the consequent part,

Manuscript received July 24, 2018; revised September 30, 2018 and December 12, 2018; accepted January 28, 2019. Date of publication February 1, 2019; date of current version November 4, 2019. (Corresponding author: Kai Meng Tay.)

Y. W. Kerk and C. P. Lim are with the Institute for Intelligent Systems Research and Innovation, Deakin University-Geelong Campus at Waurn Ponds, Geelong, Vic. 3216, Australia (e-mail: kerkyiwen@ hotmail.com; chee.lim@deakin.edu.au).

K. M. Tay is with the Faculty of Engineering, Universiti Malaysia Sarawak (UNIMAS), Kota Samarahan 94300, Malaysia (e-mail: tkaimeng@ yahoo.com).

This paper has supplementary downloadable material available at http://ieeexplore.ieee.org, provided by the author.

Color versions of one or more of the figures in this paper are available online at http://ieeexplore.ieee.org.

Digital Object Identifier 10.1109/TFUZZ.2019.2896852 is required for constructing a monotone zero order TSK-FIS (ZOTSK-FIS) model [9]-[11]. It is also important to realize that the requirements for Mamdani FIS to be monotone is more restrictive than that of ZOTSK-FIS, with the additional criterion of smooth fuzzy rules [8]. However, in a Mamdani FIS model, the fuzzy consequents of fuzzy rules that are consistent, complete, and monotone, but not smooth can be defuzzified to (and represented by) real numbers, such that they can be processed by using the ZOTSK-FIS model [13].

From the literature, Fuzzy Rule Interpolation (FRI) [14][16] focuses on methods to interpolate sparse fuzzy rules such that they are useful for tackling problems related to an incomplete fuzzy rule base. Incomplete fuzzy rules arise when human experts fail (e.g., due to fatigue) to give a complete set of fuzzy rules for constructing a knowledge-based FIS model [17], or when missing data occur in a data-driven FIS model [18]. In [19], a monotone FRI (MFRI) method was devised to form a complete and monotone fuzzy rule base from an incomplete one for ZOTSK-FIS. In addition, monotone fuzzy rule relabeling (MFRR) [9] was proposed to obtain a monotone fuzzy rule base from a nonmonotone one by relabeling the potential nonmonotone (or noisy) fuzzy rules. Nonmonotone fuzzy rules arise when human judgment errors occur [9], or due to the learning phenomenon explained in [20], where monotone data do not always produce monotone fuzzy rules. A simple solution to select a fuzzy rule among the inconsistent ones, e.g., choosing the first-appeared fuzzy rule [21], was reported to be useful for ZOTSK-FIS. Inconsistent fuzzy rules arise when inter- and intra-inconsistencies from human experts occur [22]-[25], or when the errors from data-driven algorithms occur [21], [25].

\section{A. Scope, Motivations, and Aims}

Even though mathematical conditions to ensure monotonicity of ZOTSK-FIS [9]-[11] are available, it is not clear how the fuzzy partitions at the antecedent part of a fuzzy rule base should be formulated such that the resulting ZOTSK-FIS model is always monotone. Therefore, in this paper, we introduce a new notion, i.e., the monotone fuzzy partition, that aims to provide a useful interpretation for the mathematical conditions in [1], [9]-[11]. We also derive a number of useful monotone fuzzy partitions from the current literature [1], [9]-[11].

When a monotone fuzzy partition is coupled with a consistent, complete, and monotone fuzzy rule base (which is termed as a fuzzy rule base with certain fuzzy rules in this paper), a monotone ZOTSK-FIS model can always be produced. Unfortunately, such an ideal fuzzy rule base is not always available in practice. Fuzzy rules for constructing monotone ZOTSK-FIS models are 